\title{
Scheme-Independent Stability Criteria for Difference Approximations of Hyperbolic Initial-Boundary Value Problems. I
}

\author{
By Moshe Goldberg* and Eitan Tadmor**
}

\begin{abstract}
Easily checkable sufficient stability criteria are obtained for explicit dissipative approximations to mixed initial-boundary value problems associated with the system $u_{t}=A u_{x}$ in the quarter plane $x \geqslant 0, t \geqslant 0$. The criteria are given entirely in terms of the boundary conditions for the outflow unknowns. The results imply that certain well-known boundary conditions, when used in combination with any (stable) dissipative scheme, always maintain stability.
\end{abstract}

0. Introduction. This paper provides a stability study of a wide class of difference approximations for a hyperbolic mixed initial-boundary value problem in the quarter plane $x \geqslant 0, t \geqslant 0$. The approximated differential system is $u_{t}=A u_{x}$ where $A$ is diagonal, and the inflow and outflow unknowns interact at the boundary. For the difference approximation we consider general dissipative explicit two-level schemes, with general boundary conditions which determine the boundary values in terms of outflow values at interior points. We show that such boundary conditions may have arbitrary degree of accuracy. This is included in Section 1.

In Section 2 we begin to discuss stability. We show that the entire approximation is stable if and only if the scalar components of its outflow part are stable; thus reducing the stability question to that of a scalar outflow problem. From that point on, our aim will be to obtain easily checkable sufficient stability criteria for the reduced problem. All our results are scheme-independent and are given exclusively in terms of the outflow boundary conditions. The only such result that we know of, is due to Kreiss ([3] ; see also [1]) who proved that for dissipative schemes, boundary extrapolation always maintains stability.

In the remainder of Section 2 we state our main result and consider several examples. The main result is for the scalar outflow case where the boundary conditions are translatory, i.e., determined at all boundary points by the same procedure. The result states that if the boundary conditions are generated by a solvable stable scheme, then the entire approximation is stable, independently of the interior scheme. The examples considered show that if the outflow boundary conditions are generated by oblique extrapolation, by explicit or implicit Euler schemes, or by the Box-scheme, then overall stability is assured.

Received October 10, 1977; revised April 13,1978.

AMS (MOS) subject classifications (1970). Primary 65M10; Secondary 65N10.

* The research of the first author was sponsored in part by the Air Force Office of Scientific Research, Air Force System Command, USAF, under Grant No. AFOSR-76-3046.

** The research of the second author is part of his Ph.D. dissertation and has been sponsored in part by the Air Force Office of Scientific Research (NAM) through the European Office of Aerospace Research, AFSC, United States Air Force, under Grant AFOSR 76-3035. 
In Section 3 we derive a preliminary scheme-independent result, which is an explicit interpretation of Kreiss' determinantal stability criteria [4]. This result, which seems to be of independent interest, is used in Section 4 to derive our stability criteria for the case of translatory boundary conditions.

The theoretical basis for our work was given by Kreiss [4] and by Gustafsson, Kreiss and Sundström [2]. We assume that the reader is familiar with these papers.

In a forthcoming paper we discuss the extension of our results to nondissipative and multi-level schemes.

We are grateful to Bjorn Engquist for many helpful discussions.

1. The Difference Approximation. Consider a first order hyperbolic system of partial differential equations

$$
\partial u / \partial t=A \partial u / \partial x, \quad x \geqslant 0, t \geqslant 0,
$$

where $u=\left(u^{(1)}(x, t), \ldots, u^{(n)}(x, t)\right)^{\prime}$ is the transposed vector of unknowns, and $A$ is a constant $n \times n$ Hermitian matrix of the form

$$
A=\left(\begin{array}{cc}
A^{\mathrm{I}} & 0 \\
0 & A^{\mathrm{II}}
\end{array}\right), \quad A^{\mathrm{I}}<0, A^{\mathrm{II}}>0 .
$$

Without restriction we may assume that $A$ is diagonal.

The solution of (1.1a) is uniquely determined if initial values

$$
u(x, 0)=f(x), \quad x \geqslant 0,
$$

and boundary conditions

$$
u^{\mathrm{I}}(0, t)=S u^{\mathrm{II}}(0, t)+g(t), \quad t \geqslant 0,
$$

are prescribed. Here the partition

$$
u^{\mathrm{I}}=\left(u^{(1)}, \ldots, u^{(l)}\right)^{\prime}, \quad u^{\mathrm{II}}=\left(u^{(l+1)}, \ldots, u^{(n)}\right)^{\prime}
$$

corresponds to that of $A$, and $S$ is an $l \times(n-l)$ rectangular matrix.

To solve the initial-boundary value problem (1.1) by a difference approximation we introduce a mesh-size $h=\Delta x>0, k=\Delta t>0$, such that $\lambda=k / h=$ constant. Using the standard notation $v_{\nu}(t)=v(\nu h, t)$, we approximate (1.1a) by a consistent two-sided difference scheme of the form

$$
v_{\nu}(t+k)=Q v_{\nu}(t), \quad v=r, r+1, \ldots,
$$

$$
Q=\sum_{j=-r}^{p} A_{j} E^{j}, \quad E v_{\nu}=v_{\nu+1},
$$

with initial values

$$
v_{\nu}(0)=f_{\nu}, \quad \nu=0,1,2, \ldots
$$

The $A_{j}$ are fixed diagonal $n \times n$ matrices depending on $A$ and on $\lambda$ such that $A_{-r}, A_{p}$ are nonsingular.

Throughout the paper we assume that scheme (1.2a) is dissipative, i.e., for some $\delta>0$ and positive integer $\omega$, the eigenvalues $\theta(\xi)$ of the amplification matrix 


$$
\hat{Q}(\xi)=\sum_{j=-r}^{p} A_{j} e^{i j \xi}, \quad-\pi<\xi \leqslant \pi
$$

satisfy

$$
|\theta(\xi)| \leqslant 1-\delta|\xi|^{2 \omega} .
$$

Since the $A_{j}$ are diagonal, dissipativity guarantees that the scheme (1.2a) is stable.

In order to uniquely determine the solution of (1.2), we must specify, at each time step, $r$ boundary values $v_{\mu}(t), \mu=0,1, \ldots, r-1$. For the outflow unknowns we do it by boundary conditions of the form

$$
\sum_{j=0}^{s} C_{\mu j}^{(1)} v_{\mu+j}^{\mathrm{II}}(t+k)=\sum_{j=0}^{s} C_{\mu j}^{(0)} v_{\mu+j}^{\mathrm{II}}(t), \quad \mu=0, \ldots, r-1,
$$

$C_{\mu j}$ being fixed diagonal $(n-l) \times(n-l)$ matrices. For the inflow part we use the physical boundary condition

$$
v_{0}^{\mathrm{I}}(t)=S v_{0}^{\mathrm{II}}(t)+g(t)
$$

together with $r-1$ additional conditions of the form

$$
v_{\mu}^{\mathrm{I}}(t)=\sum_{j=0}^{q} D_{\mu j} v_{j}^{\mathrm{II}}(t)+g_{\mu}(t), \quad \mu=1, \ldots, r-1,
$$

where $D_{\mu j}$ are fixed $l \times(n-l)$ matrices and the $g_{\mu}(t)$ are vectors depending on $h$ and on $g(t)$.

It is well known that using conditions of the general form (1.3), one can achieve at the boundary arbitrary degree of accuracy. We note that this is true also for conditions of type (1.4b). In fact, if accuracy of order $d$ is desired, then using the differential system and (1.1c), we find that a Taylor expansion of a smooth solution of (1.1) yields

$$
\begin{aligned}
u_{\mu}^{\mathrm{I}}(t) & =\sum_{j=0}^{d} \frac{(\mu h)^{j}}{j !} \frac{\partial^{j}}{\partial x^{j}} u^{\mathrm{I}}(0, t)+O\left(h^{d+1}\right) \\
& =\sum_{j=0}^{d} \frac{(\mu h)^{j}}{j !}\left(A^{\mathrm{I}}\right)^{-j} \frac{\partial^{j}}{\partial t^{j}} u^{\mathrm{I}}(0, t)+O\left(h^{d+1}\right) \\
& =\sum_{j=0}^{d} \frac{(\mu h)^{j}}{j !}\left(A^{\mathrm{I}}\right)^{-j}\left[S \frac{\partial^{j}}{\partial t^{j}} u^{\mathrm{II}}(0, t)+\frac{d^{j}}{d t^{j}} g(t)\right]+O\left(h^{d+1}\right) \\
& =\sum_{j=0}^{d} \frac{(\mu h)^{j}}{j !}\left(A^{\mathrm{I}}\right)^{-j}\left[S\left(A^{\mathrm{II}}\right)^{j} \frac{\partial^{j}}{\partial x^{j}} u^{\mathrm{II}}(0, t)+\frac{d^{j}}{d t^{j}} g(t)\right]+O\left(h^{d+1}\right) .
\end{aligned}
$$

Thus, (1.4b) follows upon approximating $\partial^{j} / \partial x^{j} u^{\mathrm{II}}(0, t)$ by linear combinations of $u_{0}^{\text {II }}(t), \ldots, u_{q}^{\text {II }}(t)$ of the right accuracy.

For example, if $v_{1}^{\mathrm{I}}(t)$ is required to second order of accuracy, we may use

$$
\frac{\partial u^{\mathrm{II}}(0, t)}{\partial x} \sim \frac{-3 u_{0}^{\mathrm{II}}(t)+4 u_{1}^{\mathrm{II}}(t)-u_{2}^{\mathrm{II}}(t)}{2 h}
$$




$$
\frac{\partial^{2} u^{\mathrm{II}}(0, t)}{\partial x^{2}} \sim \frac{u_{0}^{\mathrm{II}}(t)-2 u_{1}^{\mathrm{II}}(t)+u_{2}^{\mathrm{II}}(t)}{2 h^{2}},
$$

to obtain a second order accurate boundary condition of the form

$$
v_{1}^{\mathrm{I}}(t)=D_{0} v_{0}^{\mathrm{II}}(t)+D_{1} v_{1}^{\mathrm{II}}(t)+D_{2} v_{2}^{\mathrm{II}}(t)+g_{1}(t)
$$

where

$$
\begin{gathered}
D_{0}=M_{0}-3 M_{1} / 2+1 / 4 M_{2}, \quad D_{1}=2 M_{1}-1 / 2 M_{2}, \quad D_{2}=-1 / 2 M_{1}+1 / 4 M_{2}, \\
M_{j}=\left(A^{\mathrm{I}}\right)^{-j} S\left(A^{\mathrm{II}}\right)^{j}, \quad j=0,1,2, \\
g_{1}(t)=g(t)+h\left(A^{\mathrm{I}}\right)^{-1} \frac{d g}{d t}(t)+\frac{h^{2}}{2}\left(A^{\mathrm{I}}\right)^{-2} \frac{d^{2} g}{d t} .
\end{gathered}
$$

2. Statement of Main Result and Examples. The difference approximation is completely defined now by the dissipative (stable) scheme (1.2) together with the boundary conditions (1.3) (1.4), and we raise the question of overall stability in the sense of Gustafsson et al. [2, Definition 3.3].

Since the $A_{j}$ are diagonal, we can split the scheme (1.2a) into its inflow and outflow parts,

and

$$
v_{\nu}^{\mathrm{I}}(t+k)=\sum_{j=-r}^{p} A_{j}^{\mathrm{I}} v_{\nu+j}^{\mathrm{I}}(t), \quad \nu=r, r+1, \ldots,
$$

where

$$
v_{\nu}^{\mathrm{II}}(t+k)=\sum_{j=-r}^{p} A_{j}^{\mathrm{II}} v_{\nu+j}^{\mathrm{II}}(t), \quad \nu=r, r+1, \ldots,
$$

$$
A_{j}=\left(\begin{array}{cc}
A_{j}^{\mathrm{I}} & 0 \\
0 & A_{j}^{\mathrm{II}}
\end{array}\right), \quad-r \leqslant j \leqslant p,
$$

correspond to the partition of $A$. We immediately see that the outflow problem (2.2) (1.3) is self contained, while the inflow problem (2.1) (1.4) depends on the outflow part to the extent that the outflow computations provide the inhomogeneous boundary values in (1.4). Thus, stability of the entire approximation is equivalent to the following two separate questions:

(a) Stability of the inflow problem (2.1) with inhomogeneous boundary values.

(b) Stability of the outflow problem (2.2) (1.3).

Since the stability definition 3.3 of [2] gives bounds for inhomogeneous boundary values, it suffices, for the inflow problem, to consider homogeneous boundary values. But then, since the $A_{j}^{\mathrm{I}}$ are diagonal, the problem splits into $l$ independent dissipative approximations with homogeneous boundary values, which were shown by Kreiss [3, Theorem 3] to be stable independently of the basic scheme. Thus the inflow problem is stable, and it remains to consider (b). Since the $A_{j}^{\mathrm{II}}$ of (2.2) and the $C_{\mu j}$ of (1.3) are diagonal, the outflow problem in (b) splits as well and we have,

THEOREM 2.1. The entire approximation (1.2)-(1.4) is stable if and only if the $n-l$ scalar components of its outflow part are stable.

So, from now on, we may restrict the stability discussion to the following scalar 
case where we approximate an initial-value problem of the form

$$
\frac{\partial u}{\partial t}=a \frac{\partial u}{\partial x}, \quad a>0, x \geqslant 0, t \geqslant 0, u(x, 0)=f(x),
$$

by a consistent dissipative scheme

$$
v_{\nu}(t+k)=Q v_{\nu}(t), \quad \nu=r, r+1, \ldots,
$$

$$
Q=\sum_{j=-r}^{p} a_{j} E^{j}, \quad E v_{\nu}=v_{\nu+1}
$$

together with initial values

$$
v_{\nu}(0)=f_{\nu}, \quad \nu=0,1,2, \ldots,
$$

and boundary conditions

$$
\sum_{j=0}^{s} c_{\mu j}^{(1)} v_{\mu+j}(t+k)=\sum_{j=0}^{s} c_{\mu j}^{(0)} v_{\mu+j}(t), \quad \mu=0, \ldots, r-1 .
$$

Here the $a_{j}$ and the $c_{\mu j}$ are constants depending on $a$ and on $\lambda$.

Our purpose is to provide easily checkable sufficient stability criteria for approximation (2.4)(2.5) which depend entirely on the boundary conditions (2.5). Particularly effective criteria of that nature will be obtained when the boundary conditions are translatory, i.e., of the form

$$
\sum_{j=0}^{s} c_{j}^{(1)} v_{\mu+j}(t+k)=\sum_{j=0}^{s} c_{j}^{(0)} v_{\mu+j}(t), \quad \mu=0, \ldots, r-1,
$$

where the $c_{j}$ are independent of $\mu$. In this case we introduce the boundary-scheme

$$
\begin{aligned}
& T^{(1)} v_{\nu}(t+k)=T^{(0)} v_{\nu}(t), \quad \nu=0, \pm 1, \pm 2, \ldots, \\
& T^{(\alpha)} \equiv T^{(\alpha)}(E)=\sum_{j=0}^{s} c_{j}^{(\alpha)} E^{j}, \quad \alpha=0,1, \quad E v_{\nu}=v_{\nu+1},
\end{aligned}
$$

which generates (2.6) upon restriction of $v$ to the values $0,1, \ldots, r-1$.

We call the boundary scheme stable if it is stable when applied to all grid points $v h,-\infty<\nu<\infty$. We state

Theorem 2.2 (The Main Theorem). Approximation (2.4)(2.6) is stable if the boundary-scheme (2.7) is stable and if

$$
T^{(1)}(\kappa) \equiv \sum_{j=0}^{s} c_{j}^{(1)} \kappa^{j} \neq 0 \quad \forall|\kappa| \leqslant 1 .
$$

The stability criterion in this theorem is independent of the basic scheme. The proof, as well as other scheme-independent results for the translatory case, are given in Section 4.

Often, the boundary-scheme is known in advance to be stable. Thus, in applying Theorem 2.2 , it only remains to verify the solvability-condition (2.8).

If the boundary conditions are explicit, i.e., of the form 


$$
v_{\mu}(t+k)=\sum_{j=0}^{s} c_{j} v_{\mu+j}(t), \quad \mu=0, \ldots, r-1,
$$

then $T^{(1)}(\kappa) \equiv 1$. Hence (2.8) is automatically fulfilled and Theorem 2.2 reduces to

COROLLARY 2.3. If the boundary conditions (2.9) are generated by a stable scheme, then approximation (2.4)(2.9) is stable.

Example 1. Determine the boundary conditions by oblique extrapolation:

$$
v_{\mu}(t+k)=v_{\mu+1}(t), \quad \mu=0, \ldots, r-1 .
$$

Clearly, the generating boundary-scheme is explicit and unconditionally stable; so by Corollary 2.3 the stability of $(2.4)(2.10)$ is assured.

Note that (2.10) is inconsistent with the differential equation (2.3), unless $\lambda a=$ 1 , in which case (2.10) coincides with our next example.

Example 2. Let the boundary conditions be generated by the right-sided explicit Euler scheme, i.e.,

$$
v_{\mu}(t+k)=v_{\mu}(t)+\lambda a\left[v_{\mu+1}(t)-v_{\mu}(t)\right], \quad \mu=0, \ldots, r-1 .
$$

Since the basic scheme (2.4a) must satisfy the Courant-Friedrichs-Levi condition $\lambda a$ $\leqslant 1$, Euler's scheme is stable too, and by Corollary 2.3 the stability of $(2.4)(2.11)$ follows.

Example 3. Take

$$
\begin{aligned}
v_{\mu}(t+k)-\lambda a\left[v_{\mu+1}(t+k)-v_{\mu}(t+k)\right] & =v_{\mu}(t), \\
\mu & =0, \ldots, r-1,
\end{aligned}
$$

which is generated by the right-sided unconditionally stable implicit Euler scheme. To comply with Theorem 2.2 , we must have

$$
T^{(1)}(\kappa) \equiv 1-\lambda a(\kappa-1) \neq 0 \quad \forall|\kappa| \leqslant 1 .
$$

Since $\lambda a>0$, then $k$ with $|\kappa| \leqslant 1$ gives

$$
\operatorname{Re} T^{(1)}(\kappa)=1+\lambda a(1-\operatorname{Re} \kappa) \geqslant 1,
$$

so $(2.4)(2.12)$ is stable.

Example 4. We use the unconditionally stable Box-scheme to generate

$$
\begin{aligned}
& v_{\mu}(t+k)+v_{\mu+1}(t+k)-\lambda a\left[v_{\mu+1}(t+k)-v_{\mu}(t+k)\right] \\
& =v_{\mu}(t)+v_{\mu+1}(t)+\lambda a\left[v_{\mu+1}(t)-v_{\mu}(t)\right], \quad \mu=0, \ldots, r-1 .
\end{aligned}
$$

Since

$$
\operatorname{Re} T^{(1)}(\kappa)=1+\operatorname{Re} \kappa+\lambda a(1-\operatorname{Re} \kappa)>0 \quad \forall|\kappa| \leqslant 1,
$$

then by Theorem 2.1 again, (2.4)(2.13) is stable.

In view of Theorem 2.1, Examples 1-4 imply that if the boundary conditions (1.3) are generated by oblique extrapolation, explicit or implicit right-sided Euler schemes, or by the Box-scheme, then the entire approximation (1.2)-(1.4) is stable.

The boundary conditions in Examples 1-4 were studied by Gustafsson et al. [2] and by Sköllermo [5] in combination with specific 3-point basic schemes. 
3. A Preliminary Determinantal Criterion. In order to investigate the stability of (2.4)(2.5) we introduce the space $l_{2}(h)$ of all grid functions

$$
w=\left\{w_{\nu}\right\}_{\nu=0}^{\infty} \quad \text { with }\|w\|^{2} \equiv h \sum_{\nu=0}^{\infty}\left|w_{\nu}\right|^{2}<\infty .
$$

We write the approximation in operator form

$$
v(t+k)=G v(t)
$$

where $G: l_{2}(h) \rightarrow l_{2}(h)$ is uniquely determined by (2.4a) and (2.5). Following Kreiss [4, Main Theorem], we will show that $G$ has no eigenvalues $z$ with $|z| \geqslant 1$. This will suffice to assure stability both in the sense of [4] and in the sense of Definition 3.3 of [2].

To check the eigenvalues of $G$ we must adopt Kreiss' recipe in [4]: If $z$ with $|z| \geqslant 1$ is an eigenvalue, then for some nontrivial $g \in l_{2}(h), G g=z g$. Thus, by (2.4a) and (2.5), $g$ must satisfy the resolvent equation

$$
z g_{\nu}=\sum_{\nu=-r}^{p} a_{j} g_{\nu+j}, \quad \nu=r, r+1, \ldots,
$$

and the boundary relations

$$
z \sum_{j=0}^{s} c_{\mu j}^{(1)} g_{\mu+j}=\sum_{j=0}^{s} c_{\mu j}^{(0)} g_{\mu+j}, \quad \mu=0, \ldots, r-1 .
$$

The most general solution of $(3.1)$ in $l_{2}(h)$ can be written as

$$
g_{\nu}=\sum_{\alpha=1}^{k} \sum_{\beta=0}^{m} \sigma_{\alpha \beta} P_{\alpha \beta}(\nu) \kappa_{\alpha}^{\nu}, \quad \nu \geqslant 0,
$$

where $\kappa_{\alpha}=\kappa_{\alpha}(z), 1 \leqslant \alpha \leqslant k$, are the distinct roots of the characteristic equation

$$
\sum_{j=-r}^{p} a_{j} \kappa^{j}-z=0
$$

which satisfy $0<\left|\kappa_{\alpha}\right|<1$, each with multiplicity $m_{\alpha}=m_{\alpha}(z) ; P_{\alpha \beta}(\nu)$ are arbitrary polynomials in $\nu$ with $\operatorname{deg}\left[P_{\alpha \beta}(\nu)\right]=\beta$; and $\sigma_{\alpha \beta}$ are free parameters yet to be determined. Since (2.4a) is dissipative and consistent with (2.3), we use Lemma 2 and part of the proof of Lemma 7 of [4] to find that for $z$ with $|z| \geqslant 1$, Eq. (3.4) has precisely $r$ roots $\kappa$ with $0<|\kappa|<1$. Thus, $g_{\nu}$ of (3.3) depends on $r$ parameters. Substituting (3.3) in (3.2), we obtain a linear homogeneous system of $r$ equations with the $r$ unknowns $\sigma_{\alpha \beta}$. The system may take the form $J \sigma^{\prime}=0$ where $J=J(z)$ is an $r \times r$ matrix and $\sigma$ is the unknown vector. This yields

LEMмA 3.1 [4, LEMmA 3]. $z$ with $|z| \geqslant 1$ is an eigenvalue of $G$ if and only if $\operatorname{det} J(z) \neq 0$.

Going through the above process, we make a particular choice of the polynomials $P_{\alpha \beta}(\nu)$ that leads to an explicit expression of $J$ which later proves useful. We choose

$$
P_{\alpha \beta}(\nu)=\kappa_{\alpha}^{-\beta} \beta !\left(\begin{array}{l}
\nu \\
\beta
\end{array}\right) .
$$

Inserting (3.5) in (3.3) and then in (3.2), we obtain 


$$
\sum_{\alpha=1}^{k} \sum_{\beta=0}^{m} \sum_{j=0}^{s}\left[z c_{\mu j}^{(1)}-c_{\mu j}^{(0)}\right] \beta !\left(\begin{array}{c}
\mu+j \\
\beta
\end{array}\right) \kappa_{\alpha}^{\mu+j-\beta} \sigma_{\alpha \beta}=0, \quad \mu=0, \ldots, r-1,
$$

which constitutes the system $J \sigma^{\prime}=0$.

At this point we associate with the boundary conditions (2.5) a set of polynomial boundary-functions

$$
b_{\mu}(z, \kappa)=\sum_{j=0}^{s}\left[z c_{\mu j}^{(1)}-c_{\mu j}^{(0)}\right] \kappa^{\mu+j}, \quad \mu=0, \ldots, r-1 .
$$

Since

$$
\frac{\partial^{\beta} b_{\mu}(z, \kappa)}{\partial \kappa^{\beta}}=\sum_{j=0}^{s}\left[z c_{\mu j}^{(1)}-c_{\mu j}^{(0)}\right] \beta !\left(\begin{array}{c}
\mu+j \\
\beta
\end{array}\right) \kappa^{\mu+j-\beta},
$$

then system (3.6) may be written as

$$
\sum_{\alpha=1}^{k} \sum_{\beta=0}^{m} \frac{\partial^{\beta} b_{\mu}\left(z, \kappa_{\alpha}\right)}{\partial \kappa_{\alpha}^{\beta}} \sigma_{\alpha \beta}=0, \quad \mu=0, \ldots, r-1 .
$$

Thus, the coefficient matrix $J$ takes the form

$$
J=\left[B\left(z, \kappa_{1}, m_{1}\right), \ldots, B\left(z, \kappa_{k}, m_{k}\right)\right],
$$

where $B\left(z, \kappa_{\alpha}, m_{\alpha}\right), 1 \leqslant \alpha \leqslant k$, are $r \times m_{\alpha}$ blocks given by

$$
\begin{aligned}
& B\left(z, \kappa_{\alpha}, m_{\alpha}\right) \\
& =\left[\left[\begin{array}{c}
b_{0}(z, \kappa) \\
b_{1}(z, \kappa) \\
\vdots \\
b_{r-1}(z, \kappa)
\end{array}\right], \frac{\partial}{\partial \kappa}\left[\begin{array}{c}
b_{0}(z, \kappa) \\
b_{1}(z, \kappa) \\
\vdots \\
b_{r-1}(z, \kappa)
\end{array}\right], \cdots, \frac{\partial^{m_{\alpha}-1}}{\partial m^{m^{-1}}}\left[\begin{array}{c}
b_{0}(z, \kappa) \\
b_{1}(z, \kappa) \\
\vdots \\
b_{r-1}(z, \kappa)
\end{array}\right]\right]_{\kappa=\kappa}
\end{aligned}
$$

Defining a partition of $r$ to be any set of positive integers $\left\{m_{i}\right\}_{i=1}^{N}$ which satisfies $m_{1}+\cdots+m_{N}=r$, we state

THEOREM 3.2. Approximation (2.4)(2.5) is stable if for every $z$ with $|z| \geqslant 1$, every partition $\left\{m_{i}\right\}_{i=1}^{N}$ of $r$, and every set of distinct values $\left\{\kappa_{i}\right\}_{i=1}^{N}$ with $0<\left|\kappa_{i}\right|<1$,

$$
\operatorname{det}\left[B\left(z, \kappa_{1}, m_{1}\right), \ldots, B\left(z, \kappa_{N}, m_{N}\right)\right] \neq 0 .
$$

Proof. Take an arbitrary $z_{0}$ with $\left|z_{0}\right| \geqslant 1$ and let $\kappa_{\alpha}\left(z_{0}\right), 1 \leqslant \alpha \leqslant k$, be the distinct roots of (3.4), each with multiplicity $m_{\alpha}\left(z_{0}\right)$. Since $\left\{m_{\alpha}\left(z_{0}\right)\right\}_{\alpha=1}^{k}$ is a partition of $r$, then (3.10) holds for our $z_{0}, \kappa_{\alpha}\left(z_{0}\right)$ and $m_{\alpha}\left(z_{0}\right)$. Thus, by Lemma 3.1, $z_{0}$ is not an eigenvalue of $G$, and by Kreiss' Main Theorem in [4], stability follows.

Since the determinant in (3.10) depends entirely on the boundary-functions (3.7), Theorem 3.2 is scheme-independent. Thus, in applying the theorem, one avoids the inherent difficulty of solving the characteristic equation (3.4).

4. Translatory Boundary Conditions. In this section we return to consider approximation $(2.4)(2.6)$ where the boundary conditions are translatory.

The boundary-functions associated with (2.6) are 


$$
b_{\mu}(z, \kappa)=\sum_{j=0}^{s}\left(z c_{j}^{(1)}-c_{j}^{(0)}\right) \kappa^{\mu+j}, \quad \mu=0, \ldots, r-1 .
$$

Thus, writing $b(z, \kappa) \equiv b_{0}(z, \kappa)$, we have

$$
b_{\mu}(z, \kappa)=\kappa^{\mu} b(z, \kappa), \quad \nu=0, \ldots, r-1 .
$$

Consequently, if $\left\{m_{i}\right\}_{i=1}^{N}$ is a partition of $r$, then the $r \times r$ matrix

$$
\left[B\left(z, \kappa_{1}, m_{1}\right), \ldots, B\left(z, \kappa_{N}, m_{N}\right)\right]
$$

in (3.10) is given by the $r \times m_{i}$ blocks

$B\left(z, \kappa_{i}, m_{i}\right)$

$$
=\left[\left[\begin{array}{c}
b(z, \kappa) \\
\kappa b(z, \kappa) \\
\vdots \\
\kappa^{r-1} b(z, \kappa)
\end{array}\right], \frac{\partial}{\partial \kappa}\left[\begin{array}{c}
b(z, \kappa) \\
\kappa b(z, \kappa) \\
\vdots \\
\kappa^{r-1} b(z, \kappa)
\end{array}\right], \cdots, \frac{\partial^{m_{i}-1}}{\partial m^{m_{i}-1}}\left[\begin{array}{c}
b(z, \kappa) \\
\kappa b(z, \kappa) \\
\vdots \\
\kappa^{r-1} b(z, \kappa)
\end{array}\right]\right]_{\kappa=\kappa_{i}}
$$

The fact that (4.1) is determined now by the single boundary-function $b(z, \kappa)$, implies the following significant simplification of Theorem 3.2.

THEOREM 4.1. Approximation (2.4)(2.6) is stable if for every $z$ with $|z| \geqslant 1$ and $\kappa$ with $0<|\kappa|<1$, we have

$$
b(z, \kappa) \equiv \sum_{j=0}^{s}\left(z c_{j}^{(1)}-c_{j}^{(0)}\right) \kappa^{j} \neq 0 .
$$

Proof. Take an arbitrary $z$ with $|z| \geqslant 1$, a partition $\left\{m_{i}\right\}_{i=1}^{N}$ of $r$, and distinct values $\kappa_{i}, 1 \leqslant i \leqslant N$, with $0<\left|\kappa_{i}\right|<1$. In order to prove stability, it suffices, by Theorem 3.2 , to verify (3.10). For this purpose, let

$$
\sum_{\mu=0}^{r-1} \gamma_{\mu}\left[\begin{array}{c}
\kappa_{1}^{\mu} b\left(z, \kappa_{1}\right) \\
\vdots \\
\partial^{m N^{-1}\left[\kappa_{N}^{\mu} b\left(z, \kappa_{N}\right)\right] / \partial \kappa_{N}^{m_{N}}}
\end{array}\right]^{\prime}=0
$$

be a vanishing linear combination of the rows of (4.1). The vector relation in (4.3) consists of $r$ scalar equations

$$
\left\{\sum_{\mu=0}^{r-1} \gamma_{\mu} \frac{\partial^{j}}{\partial \kappa^{j}}\left[\kappa^{\mu} b(z, \kappa)\right]\right\}_{\kappa=\kappa_{i}}=0, \quad 1 \leqslant i \leqslant N, 0 \leqslant j \leqslant m_{i}-1,
$$

which we write as

$$
\frac{\partial^{j}}{\partial \kappa^{j}}\left\{b(z, \kappa)\left[\sum_{\mu=0}^{r-1} \gamma_{\mu} \kappa^{\mu}\right]\right\}_{\kappa=\kappa_{i}}=0, \quad 1 \leqslant i \leqslant N, 0 \leqslant j \leqslant m_{i}-1 .
$$

Since $0<\left|\kappa_{i}\right|<1$, then by hypothesis, 


$$
[b(z, \kappa)]_{\kappa=\kappa_{i}} \neq 0, \quad 1 \leqslant i \leqslant N .
$$

Thus, expanding (4.4) by Leibniz' rule and using induction on $j \geqslant 0$, we find that the sum in (4.4) has vanishing derivatives at $\kappa=\kappa_{i}$, i.e.,

$$
\frac{d^{j}}{d \kappa^{j}}\left[\sum_{\mu=0}^{r-1} \gamma_{\mu} \kappa^{\mu}\right]_{\kappa=\kappa_{i}}=0, \quad 1 \leqslant i \leqslant N, 0 \leqslant j \leqslant m_{i}-1 .
$$

We conclude that the polynomial

$$
P(\kappa) \equiv \sum_{\mu=0}^{r-1} \gamma_{\mu} \kappa^{\mu}
$$

which is of degree $r-1$ at most, has $r$ roots; $\kappa_{i}, 1 \leqslant i \leqslant N$, each with multiplicity $m_{i}$. Hence, $P(\kappa) \equiv 0$ and the coefficients $\gamma_{\mu}$ must vanish. By (4.3), therefore, the rows of (4.1) are linearly independent, so (3.10) holds, and stability follows.

Before turning to the proof of the Main Theorem (Theorem 2.2) we note that since Theorem 4.1 applies also to single-leveled boundary conditions, we can immediately obtain the following result of Kreiss [3] (see also [1]): Let the boundary conditions (2.6) be determined by extrapolation of arbitrary degree $s-1$, i.e.,

$$
\sum_{j=0}^{s}\left(\begin{array}{l}
s \\
j
\end{array}\right)(-1)^{j} v_{\mu+j}(t)=0, \quad \mu=0, \ldots, r-1 .
$$

The associated boundary-function is

$$
b(\kappa)=-\sum_{j=0}^{s}\left(\begin{array}{l}
s \\
j
\end{array}\right)(-1)^{j_{k} j}=-(1-\kappa)^{s} .
$$

Thus, $b(\kappa) \neq 0$ for $0<|\kappa|<1$, and by Theorem $4.1,(2.4)(4.5)$ is stable.

Proof of Theorem 2.2. The amplification factor $\hat{T}(\xi)$ of the boundary-scheme (2.7) is given by

$$
\hat{T}(\xi)=\hat{T}^{(0)}(\xi) / \hat{T}^{(1)}(\xi), \quad \hat{T}^{(\alpha)}(\xi)=T^{(\alpha)}\left(e^{i \xi}\right), \quad \alpha=0,1 .
$$

By $(2.8)$ we have $\hat{T}^{(1)}(\xi) \neq 0$; thus, $\hat{T}(\xi)$ is well defined. Since the boundary-scheme is stable, then $|\hat{T}(\xi)| \leqslant 1$, hence

$$
\left|\hat{T}^{(0)}(\xi)\right| \leqslant\left|\hat{T}^{(1)}(\xi)\right| \neq 0 \quad \forall \xi .
$$

The boundary-function associated with (2.6) satisfies

$$
b(z, \kappa)=\sum_{j=0}^{s}\left(z c_{j}^{(1)}-c_{j}^{(0)}\right) \kappa^{j}=z T^{(1)}(\kappa)-T^{(0)}(\kappa) .
$$

So, for $|z|>1$, we use (4.6) to find that

$$
\begin{aligned}
\left|b\left(z, e^{i \xi}\right)\right| & =\left|z T^{(1)}\left(e^{i \xi}\right)-T^{(0)}\left(e^{i \xi}\right)\right|=\left|z \hat{T}^{(1)}(\xi)-\hat{T}^{(0)}(\xi)\right| \\
& \geqslant|z| \cdot\left|\hat{T}^{(1)}(\xi)\right|-\left|\hat{T}^{(0)}(\xi)\right|>0 .
\end{aligned}
$$

That is, the equation

$$
b(z, \kappa)=0 \quad \text { with }|z|>1,
$$


has no roots $\kappa$ with $|\kappa|=1$. Since the roots $\kappa$ of (4.7) are continuous functions of $z$, we conclude that the number of $\kappa$ with $|\kappa|<1$ is fixed for $|z|>1$, and can be determined by considering large values of $|z|$. Writing (4.7) in the form

$$
T^{(1)}(\kappa)-z^{-1} T^{(0)}(\kappa)=0,
$$

we let $|z| \rightarrow \infty$ and use (2.8) to find that (4.7) has no roots in the unit disc. In other words, if $|z|>1$ and $b(z, \kappa)=0$, then $\kappa$ must satisfy $|\kappa|>1$. By continuity, therefore, if $|z| \geqslant 1$ and $b(z, \kappa)=0$, then $|\kappa| \geqslant 1$; i.e.,

$$
b(z, \kappa) \neq 0, \quad \forall|z| \geqslant 1,|\kappa|<1 .
$$

This implies (4.2), and Theorem 4.1 completes the proof.

Department of Mathematics

University of California

Los Angeles, California 90024

Department of Mathematical Sciences

Tel Aviv University

Tel Aviv, Israel

1. M. GOLDBERG, "On a boundary extrapolation theorem by Kreiss," Math. Comp., v. 31, 1977 , pp. 469-477.

2. B. GUSTAFSSON, H.-O. KREISS \& A. SUNDSTRÖM, "Stability theory of difference approximations for mixed initial boundary value problems. II," Math. Comp., v. 26, 1972, pp. 649-686.

3. H.-O. KREISS, "Difference approximations for hyperbolic differential equations," Numerical Solution of Partial Differential Equations (Proc. Sympos., Univ. of Maryland, College Park, Maryland, 1965), Academic Press, New York, 1966, pp. 51-58.

4. H.-O.KREISS, "Stability theory for difference approximations of mixed initial boundary value problems. I," Math. Comp., v. 22, 1968, pp. 703-714. MR 39 \#2355.

5. G. SKÖLLERMO, How the Boundary Conditions Affect the Stability and Accuracy of Some Implicit Methods for Hyperbolic Equations, Report \#62, 1975, Dept. of Computer Science, Uppsala University. 